\title{
Miopia aguda e glaucoma de ângulo fechado associados ao uso de topiramato em paciente jovem: relato de caso
}

\author{
Acute myopia and angle closure glaucoma associated with \\ topiramate use in a young patient: case report
}

\author{
Mariana Neves Brandão ${ }^{1}$ \\ Ivana Coutinho Fernandes ${ }^{2}$ \\ Flávia Fernanda Oliveira Barradas ${ }^{3}$ \\ Juliana Franca Machado ${ }^{4}$ \\ Mariane Tavares de Oliveira ${ }^{5}$
}

\section{RESUMO}

O glaucoma agudo de ângulo fechado pode ser secundário, dentre outras causas, ao uso de medicações sistêmicas, como o anticonvulsivante topiramato. Esse trabalho descreve o caso de uma paciente jovem com quadro agudo bilateral de miopia e glaucoma de ângulo fechado induzidos por terapia com topiramato para prevenção de crises de enxaqueca, fazendo relação com casos semelhantes descritos na literatura e revisão bibliográfica referentes à entidade.

Descritores: Glaucoma/epidemiologia; Glaucoma de ângulo fechado; Glaucoma de ângulo fechado/etiologia; Glaucoma de ângulo fechado/induzido quimicamente; Glaucoma de ângulo fechado/terapia; Anticonvulsivos/efeitos adversos; Relatos de casos [Tipo de pubicação]

\section{INTRODUÇÃO}

O topiramato é um agente antiepiléptico relativamente novo, classificado como monossacarídeo sulfamato substituído. Acredita-se que a droga produza seus efeitos basicamente a partir dos seguintes mecanismos de ação ${ }^{(1)}$ : diminuição da frequência da reativação dos canais de sódio, potencialização da atividade do neurotransmissor inibitório GABA (ácido gama aminobutírico) e inibição do neurotransmissor excitatório glutamato. O medicamento ainda tem propriedade inibidora de algumas isoenzimas da anidrase carbônica, efeito este, muito mais fraco que o da acetazolamida.

O uso do topiramato tem demonstrado resultados satisfatórios em casos de epilepsia de difícil controle, além de prevenção e tratamento de crises de enxaqueca ${ }^{(1-3)}$, distúrbios bipolares do humor e até obesidade. Diversos efeitos adversos têm sido atribuídos à terapêutica com topiramato, tanto em crianças quanto adultos, geralmente no primeiro mês do tratamento e que podem tornar-se potencialmente $\operatorname{graves}^{(4)}$.

As alterações oftalmológicas não são comuns, sendo que o mais alarmante é o quadro de miopia aguda associada a glaucoma de ângulo fechado bilateral, que geralmente regride com a interrupção da droga ${ }^{(2-5)}$. O processo acontece por efusão uveal devido a edema do corpo ciliar, com consequente anteriorização do diafragma irido-cristalinano, levando à diminuição da profundidade da câmara anterior e fechamento do seio camerular sem bloqueio pupilar. Já foram relatados casos de hemianopsia unilateral e maculopatia irreversível associadas ao uso do topiramato ${ }^{(1)}$.

A seguir é descrito o caso de uma paciente jovem com glaucoma bilateral de ângulo fechado secundário a tratamento para enxaqueca com topiramato. 


\section{RELATO DE CASO}

Paciente branca, sexo feminino, 32 anos de idade, procurou atendimento extra no ambulatório do Instituto Brasileiro de Oftalmologia e Prevenção da Cegueira (IBOPC), em Salvador, com queixa de dor ocular e diminuição importante da acuidade visual em ambos os olhos (AO) há cerca de 24 horas. $\mathrm{Na}$ madrugada anterior procurou serviço de emergência clínica, onde foi prescrito colírio de brinzolamida $1 \%\left(\right.$ Azopt $\left.^{\circledR}\right)$ de 4 em 4 horas e dipirona oral. A paciente havia sido examinada no próprio IBOPC dois meses antes, onde foi evidenciada emetropia e quadro de olho seco, sendo prescrito lubrificante ocular.

No momento da consulta extra a paciente referia não ter notado melhora após início da instilação da brinzolamida, doze horas antes. Relatava história de depressão e insônia, para a qual vinha em uso de Rivotril $^{\circledR}$ há cerca de um ano e uso de "analgésico (sic)" para prevenção de crises de enxaqueca há nove dias.

Ao exame oftalmológico, apresentava acuidade visual não corrigida de contar dedos a 2 metros no olho direito (OD) e 20/400 no olho esquerdo (OE), com acuidade visual bilateral para perto normal (J1). A auto-refração sem cicloplegia evidenciava $-4,50 \approx 0,50 \times 10^{\circ}$ no $\mathrm{OD}$ e $-3,50 \approx-0,50 \times 170^{\circ}$ no OE, levando à acuidade visual corrigida de 20/30 em AO. À biomicroscopia notava-se conjuntiva e córnea claras, câmara anterior (CA) muito rasa nos 2 olhos, sem sinais de reação inflamatória (Figura 1). As pupilas estavam mióticas e reagiam normalmente à luz. A tonometria foi de $28 \mathrm{mmHg}$ no OD e $26 \mathrm{mmHg}$ no OE. Ao exame com oftalmoscópio direto, foram visualizadas escavações papilares aparentemente normais, estimadas em 0,3 no OD e 0,4 no OE, sem alterações no polo posterior. Na avaliação gonioscópica com lente de Zeiss observou-se ângulo fechado em AO, que à identação vislumbrava esporão escleral em quadrante inferior do OE.

Diante do quadro descrito, foi feito diagnóstico clínico de glaucoma agudo bilateral, possivelmente mascarado pelo uso da brinzolamida. No primeiro momento, a paciente foi tratada com acetazolamida $\left(\right.$ Diamox $^{\circledR}$ ) oral $250 \mathrm{mg}$, colírios cloridrato de pilocarpina $2 \%$ e maleato de timolol $0,5 \%$. Decorrida uma hora, a paciente referia cefaléia intensa e a pressão intraocular (PIO) era de $28 \mathrm{mmHg}$ em AO. Foi suspensa a pilocarpina e optou-se pela realização de iridotomia periférica com Yag Laser em AO. Após algumas horas, a CA encontrava-se mais profunda bilateralmente e a PIO era de 22 $\mathrm{mmHg}$ no OD e $20 \mathrm{mmHg}$ no OE.

Por tratar-se de glaucoma bilateral em paciente jovem, possivelmente secundário, foi feita uma anamnese mais detalhada nesse mesmo dia, a fim de identificar provável causa desencadeante do processo. Nesse momento tivemos acesso à informação de que a medicação usada para enxaqueca tratava-se do topiramato $(50 \mathrm{mg})$. Foi feito breve estudo do mecanismo de ação da droga e seu uso suspenso imediata- mente. Evidenciamos a falta de necessidade do uso da pilocarpina e das iridotomias, que diante do quadro emergencial, acreditamos tratar-se de condutas apropriadas.

A paciente foi liberada para casa em uso de Combigan ${ }^{\circledR} \mathrm{e}$ orientada a retornar após 48 horas ou se houvesse piora do quadro. Após dois dias, a acuidade visual não corrigida era de 20/60 no OD e 20/40 no OE, e a PIO de 15 mmHg em AO. No sexto dia após o primeiro atendimento, a paciente encontrava-se assintomática, com acuidade visual sem correção de 20/25 em AO e PIO de 8 e 9 mmHg no OD e OE, respectivamente. Foi feita ultra-sonografia ocular que evidenciou descolamento parcial de vítreo posterior em AO. Era possível identificar faixa ciliar na gonioscopia de ambos os olhos. À biomicroscopia via-se CA profunda e iridotomias pérvias em AO. A fundoscopia mantinha-se sem alterações.

O colírio antiglaucomatoso foi suspenso e a paciente reavaliada com quinze e trinta dias, apresentando apenas queixa de visão de moscas volantes, sem alterações biomicroscópicas, tonométricas, gonioscópicas ou fundoscópicas. Foi solicitada uma biomicroscopia ultrassônica bilateral e a paciente referenciada ao neurologista para busca de novo tratamento pra enxaqueca, mas não mais retornou.

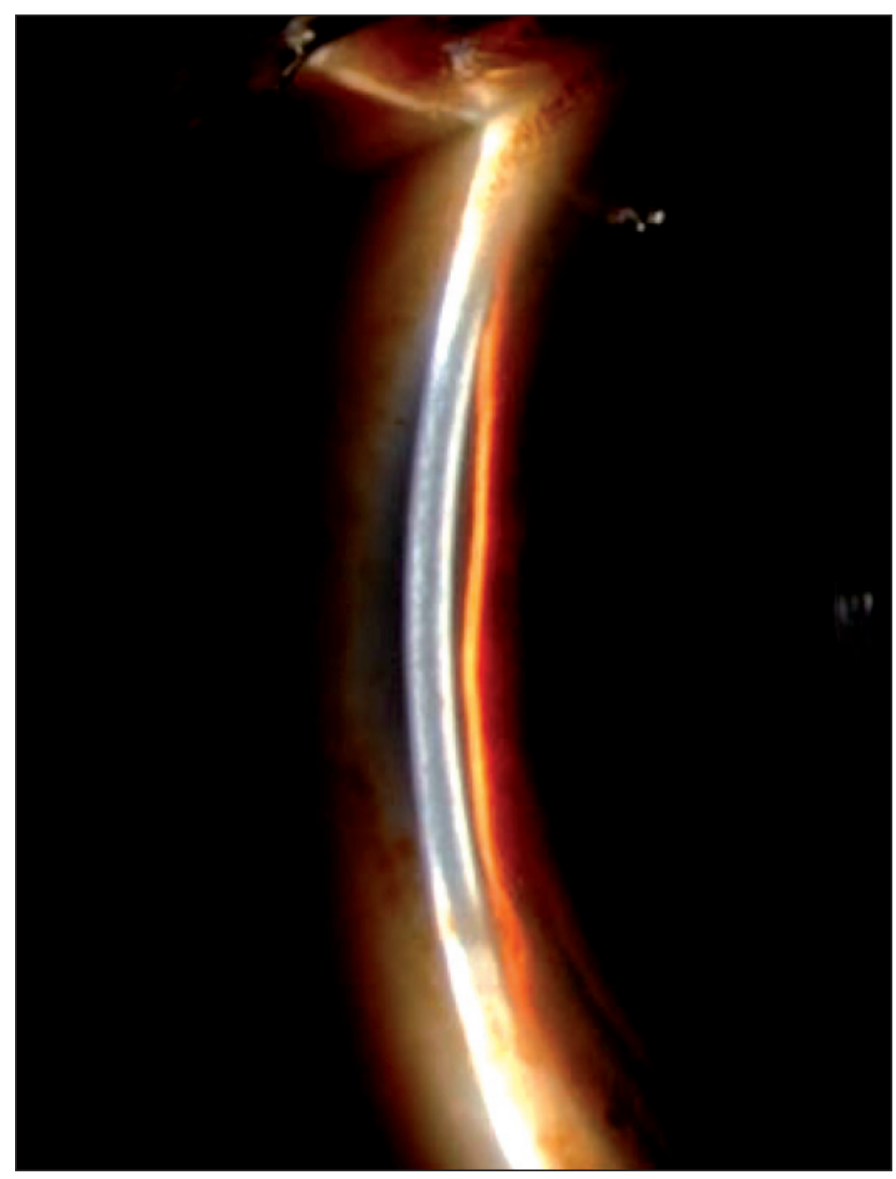

Figura 1 - Biomicroscopia do olho direito evidenciando câmara anterior rasa no momento do primeiro exame 


\section{DISCUSSÃO}

O glaucoma de ângulo fechado pode ser primário ou secundário, e este, agudo ou crônico $^{(6)}$. Devido à diversidade de mecanismos fisiopatológicos relacionados a cada uma dessas entidades, faz-se necessário, além do tratamento do glaucoma, a busca pela identificação de sua etiologia, a fim de ser tomada melhor conduta terapêutica para cada caso.

Enquanto o glaucoma primário de ângulo fechado é mais comum entre pacientes femininos, idosos e asiáticos ${ }^{(7)}$, o glaucoma secundário não apresenta predileção por população específica, já que pode ser desencadeado por inúmeras situações distintas, como traumatismos, uveítes, associação com anomalias congênitas e uso de determinadas medicações, entre outras ${ }^{(6)}$.

Os pacientes com maior risco de glaucoma secundário ao uso de medicações sistêmicas não esteroidais são aqueles com olhos pequenos, hipermetrópicos, com CA rasa, desproporção entre cristalino e segmento anterior e aqueles com ângulo estreito ${ }^{(6)}$.

Já foi identificada miopia aguda induzida por derivados da sulfa como acetazolamida, sulfametoxazol-trimetropim, prometazina, espironolactona e outros ${ }^{(3,8-9)}$. Recentemente, têm sido relatados alguns poucos casos na literatura mundial relacionando quadro de miopia aguda e glaucoma bilateral de ângulo fechado com o uso de topiramato ${ }^{(2,5,8-10)}$, como o que foi descrito nesse trabalho.

A não identificação do agente etiológico no momento do primeiro exame nos levou a fazer uso de agente miótico e iridotomias periféricas, que não seriam indicados nessa situação e poderiam ter agravado o caso, já que se acredita que a causa do glaucoma seja por efusão uveal sem bloqueio pupi$\operatorname{lar}^{(2,10)}$. Diagnosticado precocemente, o quadro clínico é totalmente reversível, necessitando para tanto, apenas a suspensão do topiramato e auxílio de colírios antiglaucomatosos para evitar que a elevação da PIO se mantenha por tempo suficiente para causar lesão ao nervo óptico.

Esse caso reforçou para a equipe a necessidade de ser dada maior importância à realização minuciosa da anamnese desde o primeiro contato com o paciente e esse trabalho teve por objetivo chamar atenção para a possibilidade de que medicamentos sistêmicos e tópicos podem levar ao aumento da pressão intraocular, podendo acarretar danos irreversíveis à visão do paciente.

\section{ABSTR ACT}

Acute angle-closure glaucoma may be induced, among other causes, by therapy with systemic drugs, such as the anticonvulsant topiramate. This paper reports the case of an young patient with acute myopia and angle-closure glaucoma associated with migraine prevention with topiramate. We make a link with similar cases described in medical journals and in a bibliographic review related to this entity.

Keywords: Glaucoma/epidemiology; Angle-closure glaucoma; Angle-closure glaucoma/etiology; Angle-closure glauco$\mathrm{ma} /$ chemically induced; Angle-closure glaucoma/therapy; Anticonvulsants/adverse effects; Case reports [Publication type]

\section{REFERÊNCIAS}

1. Asensio-Sánchez VM, Torreblanca-Agüera B, Martínez-Calvo S, Calvo MJ, Rodríguez R. [Severe ocular side effects with Topamax]. Arch Soc Esp Oftalmol. 2006;81(6):345-8. Spanish.

2. Stangler F, Prietsch RF, Fortes Filho JB. Glaucoma agudo bilateral em paciente jovem secundário ao uso de topiramato: relato de caso. Arq Bras Oftalmol. 2007;70(1):133-6.

3. Chalam KV, Tillis T, Syed F, Agarwal S, Brar VS. Acute bilateral simultaneous angle closure glaucoma after topiramate administration: a case report. J Med Case Reports. 2008;2:1.

4. Cereza G, Pedrós C, Garcia N, Laporte JR. Topiramate in non-approved indications and acute myopia or angle closure glaucoma. Br J Clin Pharmacol. 2005;60(5):578-9.

5. Chen TC, Chao CW, Sorkin JA. Topiramate induced myopic shift and angle closure glaucoma. Br J Ophthalmol. 2003;87(5):648-9.

6. Almeida HG, Cohen R, editores. Glaucomas secundários. $2^{\underline{a}}$ ed. São Paulo: Roca; 2006.

7. Mello PAA, Mandia Jr C, Galvão RP,editores. 1ำ Consenso Brasileiro de Glaucoma Primário de Ângulo Fechado. Sociedade Brasileira de Glaucoma. São Paulo: PlanMark; 2006.

8. Rhee DJ, Goldberg MJ, Parrish RK. Bilateral angle-closure glaucoma and ciliary body swelling from topiramate. Arch Ophthalmol. 2001;119(11):1721-3. Comment in: Arch Ophthalmol. 2002;120(12):1775.

9. Sankar PS, Pasquale LR, Grosskreutz CL. Uveal effusion and secondary angle-closure glaucoma associated with topiramate use. Arch Ophthalmol. 2001;119(8):1210-1. Comment in: Arch Ophthalmol. 2002;120(8):1108.

10. Medeiros FA, Zhang XY, Bernd AS, Weinreb RN. Angle-closure glaucoma associated with ciliary body detachment in patients using topiramate. Arch Ophthalmol. 2003;121(2):282-5. 